\title{
Diskussion zum Vortrag von Michael Pawlik
}

\author{
Leitung: CHRISTIAN STARCK
}

STARCK:

Vielen Dank, Herr Pawlik, für diesen interessanten Vortrag, in dem ich als Staatsrechtler Neues gehört habe, zum Beispiel, dass der Bürger die Pflicht habe, die staatliche Freiheitsgarantie zu unterstützen. Ich glaube, dass wir jetzt reichlich Diskussionsstoff haben, und ich darf fragen, wer möchte etwas sagen? Herr Patzig bitte.

PATZIG:

Mir ist es auch so gegangen, dass ich diesen Ansatz mit großem Interesse angehört habe. Allerdings habe ich einen, wie ich meine, ziemlich grundsätzlichen Einwand: Man kann Ihre Ausführungen nur auf einen gewissen Teil alles dessen, was strafrechtliche Probleme aufwirft, anwenden. Zum Beispiel fällt so etwas wie Tierschutz, der ja auch strafrechtlich geschützt ist, oder Gesetze gegen Fahrlässigkeit im Umgang mit irgendwelchen gefährlichen Stoffen, etwa Bakterien oder Viren, die eine Seuche hervorrufen können, aus dem von Ihnen skizzierten Rahmen heraus. Dazu gehören Straftaten, die schwere Schäden auch bei vielen Menschen hervorrufen können. Derlei lässt sich, denke ich, nicht auf den Nenner bringen, den Sie in Ihrem Vortrag in den Vordergrund gerückt haben, nämlich dass strafbare Handlungen einen Verstoß gegen die herkömmlichen Arten und Weisen, wie wir unsere Handlungen selbst bestimmen können, darstellen. Alles, was Sie gesagt haben, ist durchaus geeignet, alle Delikte zu erfassen, durch die in der Tat die Handlungsfähigkeiten eines Menschen durch einen anderen, bis hin zum Mord, eingeschränkt werden. Aber es gibt daneben, so meine ich, noch einen sehr weiten Bereich ganz anders strukturierter strafbedrohter Handlungen, die man mit diesem Verstoß gegen die Ordnung, wie die Menschen miteinander umgehen, jedenfalls nicht auf den ersten Blick oder vielleicht auch nicht auf den zweiten Blick in Verbindung bringen kann. Also: Es scheint mir das, was Sie gesagt haben, hervorragend zuzutreffen auf einen Sektor, nicht aber auf das ganze Gebiet, auf das Strafrecht angewendet werden muss.

STARCK:

Herr Behrends.

BEHRENDS:

Meine Frage geht dahin, wie Ihr Modell einzuordnen ist: Die Idee einer Pflicht zu legalem Verhalten, deren Erfüllung ein Stück Freiheit verwirklicht, 
weswegen bei Versagen gegenüber dieser Anforderung ein Verlust an Freiheit eintritt - das erinnert doch sehr an die Forderung nach Übereinstimmung mit der volonté générale, dem Allgemeinwillen. Sie ähnelt auch HEGELschen Konstruktionen und scheint mir daher in gewisser Weise dem Verdacht einer hochspekulativen Theorie nicht ohne weiteres entzogen zu sein. Ich möchte demgegenüber darauf hinweisen, dass man das Strafrecht auch in nüchterner Parallele zum Privatrecht sehen kann. So wie es das Schutzbedürfnis gegenüber dem privatrechtlichen Bereich gibt, auf das traditionell mit Schadenersatz reagiert wird und nur noch selten mit Bußen, so gibt es anerkanntermaßen einen Bereich von Schutzgütern, bei deren Verletzung die res publica, die gemeinsame Sache, das Gemeinwohl, wie immer man das nennen will, als Inbegriff der Werte des Zusammenlebens sich tangiert fühlt und reagiert. Bei dieser Betrachtung liegt letztlich das empirische neminem laede auch dem Strafrecht zugrunde und nicht eine Idee, die letztlich der Freiheit im heutigen Sinn widerspricht. Die Freiheit ihrer Konstruktion ist ja nicht die natürliche Handlungsfreiheit, die viele rechtlich neutrale Verhaltensalternativen umfasst, sondern, wie ROUSSEAU das gesagt hat, la liberté au sens philosophique, die im Gehorsam bestehende Freiheit. Das ist mir doch etwas, wie soll ich sagen, belastet wegen der bekannten Wirkungsgeschichte dieser Konzeption und ihrer Unvereinbarkeit mit der empirischen Freiheit der freien Entfaltung. Dazu vielleicht ein paar klärende Worte.

STARCK:

Vielleicht jetzt erst einmal zwei Antworten.

PAWLIK:

Ja gern. Zunächst einmal zu Ihnen, Herr Patzig. Zwei Punkte würde ich gern herausstellen. Erstens: Man muss unterscheiden zwischen der Frage nach der Strafbegründung und derjenigen nach der Legitimität der mit Strafe bewehrten Verhaltensnormen. Die Behandlung der ersten Frage ist analytisch zu trennen von dem zweiten Problemkreis. Ich habe mich in diesem Vortrag der Themenvorgabe entsprechend auf die erste Komponente beschränkt. Ich möchte aber gerne ganz kurz auch einiges zur zweiten Komponente sagen und mich dabei auf einen Punkt konzentrieren. Freiheit ist in einem demokratischen Gemeinwesen nicht nur die Handlungsfreiheit des Bourgeois, wenn ich es so sagen darf, sondern sie hat auch eine demokratische Komponente. Es bestehen deshalb keine grundsätzlichen freiheitstheoretischen Einwände dagegen, dass das Gemeinwesen, repräsentiert durch seine demokratisch gewählten Vertreter, in gewissem Umfang auch Straftatbestände schafft, die Interessen schützen, welche nicht auf menschliche Handlungsinteressen zurückgerechnet werden können. Ich sehe nicht, dass mein Ansatz einen Anhalt dafür böte, diese zweite, demokratische Komponente zu eskamotieren.

Nun zu Ihrem Einwand, Herr Behrends. Sie meinen im Hintergrund meiner Konzeption die volonté générale zu erkennen und halten den ganzen Ansatz für hochspekulativ. Im weiteren Verlauf Ihrer Ausführungen räumen Sie dann 
freilich selbst ein, es gebe Fälle, in denen die res publica sich tangiert fühle und reagiere. Ohne Ihnen zu nahe treten $\mathrm{zu}$ wollen, scheint es mir so zu liegen, dass das, was ich ausgefuihrt habe, diesen kompakten Satz lediglich ausbuchstabiert. Mit dem Befund, dass eine demokratisch konstituierte res publica sich tangiert fühlt und reagiert - und zwar nicht irgendwie, sondern mittels der Strafe - ist noch nicht die Frage beantwortet, weshalb sie das darf. Um diese Frage $\mathrm{zu}$ beantworten, ist es meines Erachtens unumgänglich anzunehmen, dass der Bürger für das Gedeihen seiner res publica einzustehen hat, jedenfalls insoweit, als er die Begehung von Straftaten zu unterlassen hat. Dies ist, so meine ich, nur ein Ausbuchstabieren der Implikationen von Überzeugungen, die weit verbreitet sind, und insofern ist es nicht rein spekulativ. Zum Stichwort volonté générale: Bei ROUSSEAU geht der Bürger in seinem Bürgersein auf. Er verliert seine alte Identität und nimmt eine neue an. Von so etwas kann in meiner Konzeption nicht die Rede sein. Herr Patzig hatte ja sogar den Eindruck, ich hätte sozusagen nur die Bourgeois-Existenz erfasst. Die Hypostasierung des Allgemeinen ist jedenfalls nicht mein Anliegen.

STARCK:

Ich danke Ihnen. Jetzt kommt als nächste Frau Hörnle und dann melde ich schon mal Herrn Frisch an. Bitte schön, Frau Hörnle.

\section{HÖRNLE:}

Eine Frage ist: Wie legitimiert man Strafe, und in diesem Punkt finde ich Ihre Ausführungen völlig überzeugend, Herr Pawlik, da stimme ich Ihnen zu. Aber es gibt ja auch die zweite Frage: Warum betreiben wir eigentlich den ganzen Aufwand? Warum investieren wir Milliarden in die Strafverfolgungsbehörden etc.? Und diese Frage kann man mit der Antwort, weil der Täter mitverantwortlich ist, nicht beantworten. Dafür brauchen wir den Rückgriff auf handfestere Interessen. Und auch Ihre Charakterisierung des Verbrechens als Verletzung einer Mitwirkungspflicht ist sicher teilweise richtig, aber sie ist unvollständig. Strafe verletzt auch reale Menschen. Und Strafe deshalb als entprivatisiert zu bezeichnen, greift meiner Ansicht nach zu kurz. Strafe soll Rachebedürfnisse einhegen, kontrollieren, befrieden. Aber in dieser Einhegung sind das Rachebedürfnis und das Genugtuungsbedürfnis doch wesentliche Säulen des Strafrechts. Das erklärt nun wiederum nicht für sämtliche Deliktstypen, warum wir bestrafen sollen, da müsste man wieder auf die negative Generalprävention zurückgreifen. Ich weiß, dass Sie diese Form der zusammengesetzten Straftheorie als nicht befriedigend empfinden, aber ist nicht eine zusammengesetzte, zusammengeklebte Straftheorie mit all ihren Bruchstellen immer noch die bessere Erklärung als eine reduzierte Straftheorie, die hier nur Einzelteile beleuchtet?

FRISCH:

Herr Pawlik, ich stimme Ihnen in einer Reihe von Punkten zu. Das gilt einmal für die Kritik an den Präventionstheorien; es gilt zum zweiten dafür, dass 
Sie die Wiederherstellung des Rechts in den Vordergrund schieben, das würde ich auch so sehen. Und ich stimme Ihnen - drittens - auch zu, wenn Sie sagen, auf der Begründungsebene sei mit der Theorie der Spezialprävention nicht allzu viel zu erreichen, jedenfalls soweit wir die Strafebene und nicht die Maßregeln in den Blick nehmen. Nun zu Ihrem eigenen Ansatz. Sie verwenden drei Schritte, drei Begriffe auch, nämlich Mensch, Subjekt, Bürger, und Sie knüpfen an den Bürger dann eine sogenannte Mitwirkungspflicht gegenüber der Rechtsgemeinschaft. Das ist wohl der eigentliche Clou an Ihrem Ansatz, die Mitwirkungspflicht gegenüber der Rechtsgemeinschaft, aus der dann im Falle des Rechtsbruchs die Duldungspflicht folgen soll. Man kann das so sehen, aber man kann auch mehrere Fragen dazu stellen. Meine erste Frage: Warum diese Verdoppelung der Pflichten? Warum noch einmal diese zusätzliche Pflicht zu der schon bestehenden? Ich habe ja bereits ein Gesetz, das zu befolgen ist, warum noch einmal diese zusätzliche Mitwirkungspflicht? Meine zweite Frage: Läuft es nicht auf eine schlichte Behauptung hinaus, wenn Sie sagen, aus der nicht erfüllten Mitwirkungspflicht werde nun eine Duldungspflicht (den strafenden Eingriff hinzunehmen)? Ich frage hier schlicht dagegen: warum eigentlich? Ist diese von Ihnen behauptete Umwandlung einer ebenfalls behaupteten Mitwirkungs- in eine Duldungspflicht nicht im Grunde genommen nur eine komplizierte normentheoretische Verschiebung des Legitimationsproblems? Und die dritte Frage, die ich noch habe: Wie begründen Sie auf der Basis dieses Ansatzes bei Verletzung der gleichen Mitwirkungspflicht unterschiedliche Strafen?

STARCK:

Zwei Redner, fünf Fragen, bitte schön, Herr Pawlik.

PAWLIK:

Vielen Dank. Ich darf mich zunächst Ihnen, Herr Frisch, zuwenden. Nach meinem eigenen Verständnis enthält mein Vortrag nicht nur einen Clou, sondern zwei. Der eine, das ist der konstruktive Gedanke, Strafe reagiere auf eine Mitwirkungspflichtverletzung. Der zweite, und dies ist mir ebenso wichtig, ist die Aussage, dass der Legitimationsgrund der Pflicht damit noch nicht bezeichnet ist. Dieser Legitimationsgrund liegt, ich sage es ganz pauschal, im Freiheitsschutz. Das bedeutet, es wird nicht bestraft um der Pflichtwidrigkeit als solcher willen, sondern der Umfang der Pflichtverletzung bemisst sich auch wesentlich nach dem Ausmaß der Freiheitsverletzung, deswegen unterschiedliche Strafen. Warum wird aus der Mitwirkungspflicht eine Duldungspflicht? Die Antwort ist: Der Bürger hat nach meiner Theorie die Pflicht, an der Aufrechterhaltung eines Zustandes von Freiheitlichkeit mitzuwirken. Dieser Pflicht hat er zuwidergehandelt, indem er sein Verbrechen verübt hat. Seinen Beitrag zur Aufrechterhaltung des Rechtszustandes kann er deshalb nur noch erbringen, indem er duldet, dass auf seine Kosten demonstriert wird: „Wer nicht ordnungsgemäß kooperiert, der verliert Freiheit.“ Der positive Grundgedanke ist: „Wenn du pflichtgemäß handelst, trägst du das Deinige dazu bei, dass der be- 
stehende Zustand von Freiheitlichkeit - und damit indirekt auch deine eigene Freiheit - erhalten bleiben.“ Umgekehrt: „Wenn - und weil - du pflichtwidrig gehandelt hast, verlierst du Freiheit. Und das zeigen wir jetzt anhand dessen, was wir mit dir machen." Schließlich: Warum eine Verdoppelung der Pflichten? Der Grund ist ein konstruktiver: Ich habe versucht darzulegen, dass wir, wenn wir vergeltungstheoretisch argumentieren, das Schadenersatzparadigma zugrunde legen müssen und dass wir auf seiner Grundlage konstruktiv dazu gezwungen sind, das Proprium des Verbrechens in seiner Allgemeinheitsbedeutung zu erblicken. Nicht zuletzt unterstreicht diese Verdoppelung die von mir hervorgehobene Unterscheidung zwischen der konstruktiven Mitwirkungspflicht und der Legitimationsproblematik.

Nun zu Ihnen, Frau Hörnle. Sie haben unterschieden zwischen zwei Fragen: Erstens, wie legitimiert sich Strafe, und zweitens, warum betreiben wir den Aufwand? Sie mögen es für banal halten, aber ich würde zu letzterem einfach sagen: Wir betreiben den Aufwand, weil wir uns als freiheitliche Rechtsgemeinschaft nur so stabilisieren können. Dass im Einzelnen sehr viele pragmatische Erwägungen mitspielen, ist völlig selbstverständlich. Aber das war hier nicht mein Thema, sondern es ging mir nur darum zu zeigen, dass wir als Rechtsgemeinschaft gute Gründe haben, Strafe zu verhängen und damit auch den Aufwand zu betreiben, der zur Strafverhängung hinzugehört. Und dass Verbrechen an realen Menschen verübt werden, dass hinterher der reale Mensch tot ist und nicht die Rechtsgemeinschaft, ist gleichfalls selbstverständlich. Dass ich dies keineswegs übersehen habe, ergibt sich, wie ich hoffe, schon aus dem, was ich eben im Hinblick auf Herrn Frisch gesagt habe: Legitimationsgrund von Strafe ist die Verletzung von Freiheit, und wer tot ist, hat viel Freiheit verloren.

STARCK:

Jetzt kommt Herr Duttge und dann Herr Schreiber.

DUTTGE:

Ich will auf dasjenige eingehen, was auch Frau Hörnle schon angedeutet hat mit dem wunderbar plastischen Begriff der "Zusammenstückelung“ mehrerer Theorieansätze. Ich bin, lieber Herr Pawlik, ganz auf Ihrer Seite, wenn Sie überzeugend darlegen, von welch unansehlicher Gestalt sich derzeit das in sich unstimmige Strafrechtsgebäude zeigt, wie sehr es uns also darum gehen muss, eine in sich geschlossene, konsistente Gesamtkonzeption der Strafbegründung und Anwendung von Strafrecht zu entwickeln. Und gerade deshalb interessiert mich besonders, wie es Ihnen gelingen soll, die im letzten Teil Ihrer Überlegungen sehr stark betonte Spezialprävention in Ihre Konzeption zu integrieren. Wenn ich Sie richtig verstanden habe, fiel in diesem Zusammenhang u.a. auch der Begriff des „Bürgerstatus“. Aus diesem Bürgerstatus soll sich im Rahmen der Strafvollstreckung ergeben, dass wir hier im Sinne des Bundesverfassungsgerichts in besonders fürsorglicher Weise darauf $\mathrm{zu}$ achten haben, dass der Verurteilte trotz verhängter lebenslanger Freiheitsstrafe noch vor seinem biolo- 
gischen Ende wieder der Freiheit teilhaftig wird. Der Bürgerstatus ist in Ihrem Konzept aber offensichtlich zugleich auch Anknüpfungspunkt für den strafrechtlichen Zugriff als solchen. Dies dürfte um so mehr gelten, wenn Sie von einer „Mitverantwortung“ oder bürgerrechtlichen „Pflicht“ des Einzelnen im Verhältnis zur Rechtsgemeinschaft sprechen, denn damit ist unverkennbar der Straftäter angesprochen, der sich durch seine Straftat gleichsam immer auch gegenüber der Rechtsgemeinschaft schuldig gemacht hat. Das heißt also: Derselbe Status soll sowohl dazu führen, den Bürger in seiner freiheitlichen Betätigung und Entfaltung ernst und im Bedeutungsgehalt seiner tätigen Kommunikation beim Worte zu nehmen, ihn daran festzuhalten, als auch - auf der Basis desselben Begründungsansatzes - im Rahmen der Strafvollstreckung dazu, aus spezialpräventiven Gründen die eigentlich verwirkte Strafe nicht ungemildert bis zur bitteren Neige auszuschöpfen. Darin liegen, wie ich meine, zwei gegenläufige Tendenzen, die sich kaum auf denselben Leitgedanken zurückführen lassen. Fehlt da nicht ein Begründungsstück für die Berechtigung spezialpräventiver Überlegungen, und wie könnte sich dieses in das Gesamtkonzept einbinden lassen? Denn es liegt auf der Hand, dass die Gegenläufigkeit jener die Strafvollstreckungsentscheidung tragenden Wertgrundlage die Strafbegründungsentscheidung und den hier zugrunde gelegten Maßstab jedenfalls tendenziell unterlaufen wird und den erstrebten einheitlichen, von der Strafbegründung bis zur Strafvollstreckung reichenden einheitlichen legitimatorischen $\mathrm{Zu}-$ sammenhang zerschlägt.

\section{SCHREIBER:}

In Ihrer Antwort auf Herrn Frisch und jetzt auch in dem, was Herr Duttge angeführt hat, habe ich den Verdacht, dass Sie Ihren ursprünglich HEGELschen Ansatz profanisiert haben, dass Sie die primäre Bürgerpflicht konstruktiv in eine Schadenersatzpflicht umwandeln. Warum muss ich eigentlich, wenn ich meiner Bürgerpflicht nicht nachgekommen bin, Schmerzen erdulden? Warum muss ich dann bestraft werden? Das ist ja gar kein Schadenersatz. Sie ersetzen ja gar nicht den Schaden, sondern Sie fügen ein Übel zu, und da kommen doch dann Elemente der Prävention hinzu. Denn mehr als die Generalprävention kann es schlicht nicht sein, was bei Hegel die Hoheit der Verletzung des Rechts ist, dass ein Übel durch ein anderes Übel vergolten wird, das ersetzen Sie hier mit Präventionsstücken. Ich habe sehr den Verdacht, dass Sie nichts anderes bieten als eine elegant überhöhte Präventionstheorie, mit der Sie der HEGELschen Metaphysik entkommen.

STARCK:

Bitte schön, Herr Pawlik.

PAWLIK:

Ja, vielen Dank. Wenn ich an Ihren eigenen Vortrag zurückdenke, Herr Duttge, glaube ich feststellen zu können, dass wir in dem zentralen Punkt übereinstimmen: Mit der Bestrafung demonstriert die Rechtsgemeinschaft, dass sie den 
Täter als Bürger ernst nimmt. Dieser Ausgangspunkt ist meiner Auffassung nach kein genuin hegelianischer. Hegelianisch ist erst der Folgesatz, dass ich den Bürger als ein Konkret-Allgemeines begreife und nicht nur als ein Abstraktum. Das heißt, ich sehe ihn auf der einen Seite als jemanden, dem wir Unrecht und Schuld zuschreiben, aber ich berücksichtige auf der anderen Seite auch seine Fehlbarkeit. Meines Erachtens liegt darin kein prinzipieller innertheoretischer Bruch; vielmehr geht es jeweils um unterschiedliche Aspekte des einen Bürgerstatus: Auf der einen Seite geht es um die Verantwortlichkeit für das eigene Tun, die wir voraussetzen müssen, sonst könnten wir keine Handlungsfreiheit gewähren. Auf der anderen Seite geht es darum, dass wir den Täter im Anschluss an sein verantwortungswidriges Verhalten nicht einfach allein lassen, sondern ihn auf dem Weg zurück zur Legalität unterstützen. Sie mögen diese Umschreibung für etwas kitschig halten. Der ihr zugrunde liegende Gedanke aber ist es nicht: Wir sollten nach meiner Überzeugung die Begriffe nicht zu abstrakt halten.

Zu Herrn Schreiber: Selbstverständlich sind mit der Bestrafung des Täters auch präventive Reflexe verbunden. Niemand, nicht einmal der radikalste Vergeltungstheoretiker, KANT, hat jemals die Tatsache und auch die Wünschbarkeit präventiver Effekte bestritten. Mir geht es aber nicht um die verschiedenen tatsächlichen Wirkungen der Strafe, sondern darum, inwieweit diese sich in haltbare legitimationstheoretische Kategorien übersetzen lassen. Dort kommt es dann zum Schwur. Es ist eben ein Unterschied - und zwar nicht nur in der Begründung, sondern vielfach auch im Ergebnis -, ob wir die Verbrechenslehre und die Grundsätze der Strafzumessung auf der Basis präventiven Denkens oder auf der Grundlage der von mir vorgestellten Konzeption entwickeln. Leider fehlte es mir an der Zeit, in meinem Vortrag auch darauf noch näher einzugehen; deshalb bleibt meine jetzige Aussage unweigerlich etwas thesenhaft. Was die Strafzumessung angeht, so habe ich immerhin eben schon angedeutet, dass zum einen - in herkömmlicher Terminologie gesprochen - das Maß der Rechtsgutverletzung und zum anderen die Beschaffenheit der gesellschaftlichen Situation, in die hinein delinquiert wird, für die Strafzumessung entscheidend sind. Ich muss es leider bei diesen Stichworten belassen. Wichtig ist mir aber, nochmals zu unterstreichen, dass ich das, was ich Ihnen vorgetragen habe, nicht als bloße Reformulierung eines präventionstheoretischen Gedankens verstehe. Ich vertrete eine Vergeltungstheorie, aber eben eine solche, die sich nicht auf die Aussage beschränkt, wir strafen um der Gerechtigkeit willen, Punktum. Wir brauchen auch den Bezug auf unsere konkrete Gesellschaft. Den können wir nicht hinauseskamotieren. Deshalb kann es nur darum gehen, die straftheoretische Bezugnahme auf diese gesellschaftliche Begründungsbasis möglichst überzeugend vorzunehmen. 


\section{STARCK:}

Vielen Dank. Ich habe jetzt noch drei Wortmeldungen notiert und möchte dann die Liste schließen, das sind Herr Jehle, Herr Naucke und Herr MüllerDietz.

JEHLE:

Ich darf an das anknüpfen, was Herr Patzig sagte, mir scheint eben auch das Feld, das Sie mit Ihrer Theorie beackern können, relativ eng. Der präventive Gedanke ist heute schon vielfach in die Tatbestandsmäßigkeit hinein genommen. In vielen Bestimmungen des modernen Strafrechts ist die Strafbarkeit so weit vorverlagert, dass längst vor der eigentlichen Rechtsgutsverletzung die Tatbestandsmäßigkeit erfüllt ist. Ein kleines Beispiel: Wenn ich ein Hanfpflänzchen - nein, nicht ich - wenn meine Kinder oder meine Studenten ein Hanfpflänzchen auf die Fensterbank stellen, bin ich bereits im strafbaren Bereich, weit vor der eigentlichen Rechtsgutsverletzung. Der moderne Gesetzgeber nimmt die Prävention schon in die Tatbestandsmäßigkeit hinein, indem er die Tatbestandsmäßigkeit sehr weit vorverlagert. Sie entkommen diesem präventiven Gedanken auch mit Ihrer Theorie nicht.

\section{STARCK:}

Herr Naucke.

\section{NAUCKE:}

Ich habe eine Frage, eher eine Überlegung, ob Sie Ihre eigenen Gedanken nicht anstücken müssten, wenn ich nun frage, in welchem Staat wird vergolten? Das Interessante ist, dass Sie nur eine Straftheorie entwickeln, aber die Straftheorie, in neuerer Zeit jedenfalls, ist immer eine Straftheorie, die mit einem bestimmten Staat verbunden ist. Mir ist aufgefallen, dass Sie am Anfang der Debatte, für mich völlig plötzlich, die Demokratie ins Spiel bringen. Wenn Sie sagen würden, ich präsentiere eine reformulierte Vergeltungstheorie, die für eine Demokratie in ruhigen Zeiten gilt, dann würde ich mit Ihnen diskutieren. Aber wenn Sie es so allgemein lassen, wie Sie es jetzt formuliert haben, dass der Bürger seinen Beitrag zur Rechtsordnung nicht erbringt usw., dann gilt das für die NS-Zeit wie für die Republik wie für die Kaiserzeit und für das Kolonialstrafrecht, und da würde ich Ihnen aber mit großem Nachdruck widersprechen, dass das nicht geht.

STARCK:

Eine wichtige Frage. Herr Müller-Dietz, der letzte.

MÜLLER-DIETZ:

Ich fand Ihre Ausführungen, Herr Pawlik, zu Präventionstheorien weitgehend überzeugend. Wo ich Schwierigkeiten habe, da bitte ich um Nachsicht, ist die Quelle für die Mitwirkungspflicht des Bürgers. Ich habe da eine Zeitlang gedacht, das ist der Gesellschaftsvertrag, aber Sie haben die volonté générale von ROUSSEAU zurückgewiesen. Mir ist nicht ganz klar, ist es der demokratisch 
legitimierte Gesetzgeber, der die Gesetze macht, an die man sich halten muss, oder was ist der Grund dafür, dass ich eben diese Pflichten einhalten muss? Vielleicht noch eine Ergänzung: Ich habe ein bisschen vermisst, dass eigentlich bei den Opferdelikten, wo es also ein Straftatopfer gibt, die Anerkennung der Respektierung des anderen als Mensch mit seinen Rechten verletzt wird. Das geht natürlich unter, indem man von einer Entindividualisierung spricht, das führt dann eben auch zu einer Sozialisierung der Strafe.

STARCK:

Herr Pawlik bitte.

PAWLIK:

Zunächst einmal zu Herrn Naucke, weil mir das persönlich auch besonders am Herzen liegt. Ich habe versucht, deutlich zu machen, dass der Legitimationsgrund der Mitwirkungspflicht die Wahrung von Freiheitlichkeit ist. Die Theorie, die ich Ihnen präsentiert habe, ist insofern eine Theorie für den freiheitlich-demokratischen Staat, nicht eine Theorie für den Nazi-Staat. Mir geht es eben nicht um Deskription, sondern um eine Legitimationstheorie; diese hat zum konstitutiven Element den Gedanken der Freiheit. Sie können also weiterhin mit mir diskutieren, Herr Naucke.

Nun zu Ihnen, Herr Müller-Dietz. Mit der Mitwirkungspflicht zahlt der Bürger die Kosten für sein Leben in Freiheit. Wir kennen ja eine Reihe rechtlicher Mitwirkungspflichten. Wenn Sie so wollen, ich sage das jetzt ganz ungeschützt, ist die Pflicht zur Mitwirkung und die aus ihr abgeleitete Pflicht zur Duldung der Strafe eine Art erweiterter Steuerpflicht. Zur Erfüllung unserer Steuerpflicht geben wir Geld, damit der Staat die Polizei und die Gerichte bezahlt. Aber so viel Polizei, so viele Gerichte kann es gar nicht geben, wenn wir uns ansonsten benehmen würden wie die Teufel. Wir müssen also auch in unserem persönlichen Verhalten die Steuer zahlen für unser Leben in Freiheit.

Nun zu dem, was Herr Jehle angesprochen hat. Ich habe schon in meiner Antwort auf Herrn Patzig betont, dass die Frage der Straflegitimation eine andere ist als die Frage der Legitimation der Normen, deren Verletzung der Gesetzgeber mit Strafe belegt. Sie haben vollkommen Recht, Herr Jehle, wir erleben eine Auflösung dessen, was man noch mit halbwegs gutem Gewissen als klassisches Strafrecht bezeichnen kann; was sich abzeichnet, ist eine Verschmelzung von Strafrecht und Polizeirecht zu einem neuartigen Sicherheitsrecht. Es ist ganz selbstverständlich, dass dies sich auch auf die Deutung von Strafe auswirken kann. Ob wir dann nämlich noch mit dem traditionellen $\mathrm{Pa}-$ thos von Schuld sprechen können, das möchte ich mit einem großen Fragezeichen versehen. Ich bin in meinem Vortrag von einem Begriff des Strafrechts ausgegangen, der dem herkömmlichen Verständnis der Strafrechtsdogmatik im Wesentlichen entspricht. Das war selbstverständlich eine Idealisierung, und Sie mögen sie als zu weitgehend ansehen. Aber es ist ja immerhin ganz nützlich, anhand eines solchen Maßstabs zu sehen, wie weit wir bereits abgedriftet sind. Insgesamt würde ich Ihrer Analyse also überhaupt nicht widersprechen, son- 
dern möchte auch meinerseits unterstreichen, dass das, was wir in vielen Fällen praktizieren, nur noch in einem formellen und kaum noch in einem materiellen Sinne Strafrecht ist.

STARCK:

Vielen Dank. 